\title{
Adjoint Sensitivity Computations for an Embedded-Boundary Cartesian Mesh Method and CAD Geometry
}

\author{
Marian Nemec ${ }^{1}$ and Michael J. Aftosmis ${ }^{2}$ \\ 1 ELORET Corp., Moffett Field, CA 94035, USA mnemec@mail.arc.nasa.gov \\ 2 NASA Ames Research Center, Moffett Field, CA 94035, USA maftosmis@maílarc.nasa.gov
}

Key words: shape optimization, adjoint, Cartesian mesh

\section{Introduction}

Cartesian-mesh methods [1] are perhaps the most promising approach for addressing the issues of flow solution automation for aerodynamic design problems. In these methods, the discretization of the wetted surface is decoupled from that of the volume mesh. This not only enables fast and robust mesh generation for geometry of arbitrary complexity. but also facilitates access to geometry modeling and manipulation using parametric Computer-Aided Design (CAD) tools. Our goal is to combine the automation capabilities of Cartesian methods with an efficient computation of design sensitivities. We address this issue using the adjoint method, where the computational cost of the design sensitivities, or objective function gradients, is essentially independent of the number of design variables. In previous work $[2,3]$, we presented an accurate and efficient algorithm for the solution of the adjoint Euler equations discretized on Cartesian meshes with embedded, cut-cell boundaries. Novel aspects of the algorithm included the computation of surface shape sensitivities for triangulations based on parametric-CAD models and the linearization of the coupling between the surface triangulation and the cut-cells.

The objective of the present work is to extend our adjoint formulation to problems involving general shape changes. Central to this development is the computation of volume-mesh sensitivities to obtain a reliable approximation of the objective function gradient. Motivated by the success of mesh-perturbation schemes commonly used in body-fitted unstructured formulations, we propose an approach based on a local linearization of a mesh-perturbation scheme similar to the spring analogy. This approach circumvents most of the difficulties that arise due to non-smooth changes in the cut-cell layer as the boundary shape evolves and provides a consistent approximation to the exact gradient of the discretized objective function. A detailed gradient accuracy study is presented to verify our approach. Thereafter, we focus on a shape optimization problem for an Apollo-like reentry capsule. The optimigation seeks to enhance the lift-to-drag ratio of the capsule by modifying the shape of its heat-shield in conjunction with a center-of-gravity (c.g.) offset. This multipoint and multi-objective optimization problem is used to demonstrate the overall effectiveness of the Cartesian adjoint method for addressing the issues of complex aerodynamic design. This abstract presents only a brief outline of the numerical method and results; full details wilb be given in the final paper.

\section{Problem Formulation}

The spatial discretization of the three-dimensional Euler equations uses a second-order accurate finite volume method with weak imposition of boundary conditions, resulting in a discrete system of equations

$$
R(Q, M)=0
$$

where $Q=\left[\bar{Q}_{1}, \bar{Q}_{2}, \ldots, \bar{Q}_{N}\right]^{\top}$ is the discrete solution vector for all $N$ cells of a given mesh $M$, and $R$ is the flux residual vector. The influence of a shape design variable, $X$, on the residuals is implicit via the computational mesh $M=f[T(X)]$, where $T$ denotes a surface triangulation of the CAD model. Hence, the gradient of a. discrete objective function $\mathcal{J}(X, M, Q)$ is given by 


$$
\frac{\mathrm{d} \mathcal{J}}{\mathrm{dX}}=\frac{\partial \mathcal{J}}{\partial X}+\underbrace{\frac{\partial \mathcal{J}}{\partial M} \frac{\partial M}{\partial T} \frac{\partial T}{\partial X}}_{\mathrm{A}}-\psi^{\mathrm{T}}(\frac{\partial R}{\partial X}+\underbrace{\frac{\partial R}{\partial M} \frac{\partial M}{\partial T} \frac{\partial T}{\partial X}}_{B})
$$

where the vector $\psi$ represents adjoint variables given by the adjoint equation, for details see [3]. The focus of this work is on the evaluation of the partial dexivative terms $A$ and $B$ in $E q .2$, which represent the differentiation of the objective function and residual equations with respect to design variables that alter the surface shape.

\section{Results}

This example targets the optimization of a heat-shield shape for a reentry capsule. The objective of the optimization is to enhance the lift-to-drag ratio $(L / D)$ of the capsule, thereby improving its trajectory control for landing-site selection, and reducing the reentry load factor and heat rates. We consider a two-point optimization problem where the design Mach numbers are 10 and 25. High-temperature effects are approximated by the use of an "effective" ratio of specific heats, $\gamma$, at each design point. We use $\gamma=1.231$ and $\gamma=1.125$, respectively. The target value of $L / D$ at each design point is set to 0.4 . This value is based on the aerodynamic characteristics of the symmetric Apollo capsule, which attained an $L / D$ of 0.3 using a c.g. offset. We use a similar c.g.offset in the present study. The parametric-CAD model of the capsule is shown on the left side of Fig. 1. The heat-shield shape is controlled by a B-spline parameterization of its center-line. The design variables are associated with three B-spline control points near the shoulder of the capsule. The pitch stability of the capsule, i.e., trim and negative $C_{m_{\alpha}}$ slope, is ensured by introducing penalty terms in the objective function. The angle of incidence at each design point is used to enforce the pitching-moment constraints, resulting in a total of five design variables. In this abstract, the gradients are computed using finite differences. The results are summarized by the two sets of images on the right side of Fig. 1, which shows pressure contours of the baseline and optimal designs at the two operating conditions. The $L / D$ is increased from 0.37 to 0.4 in 15 design iterations and the gradient is reduced by roughly 2.5 orders of magnitude.
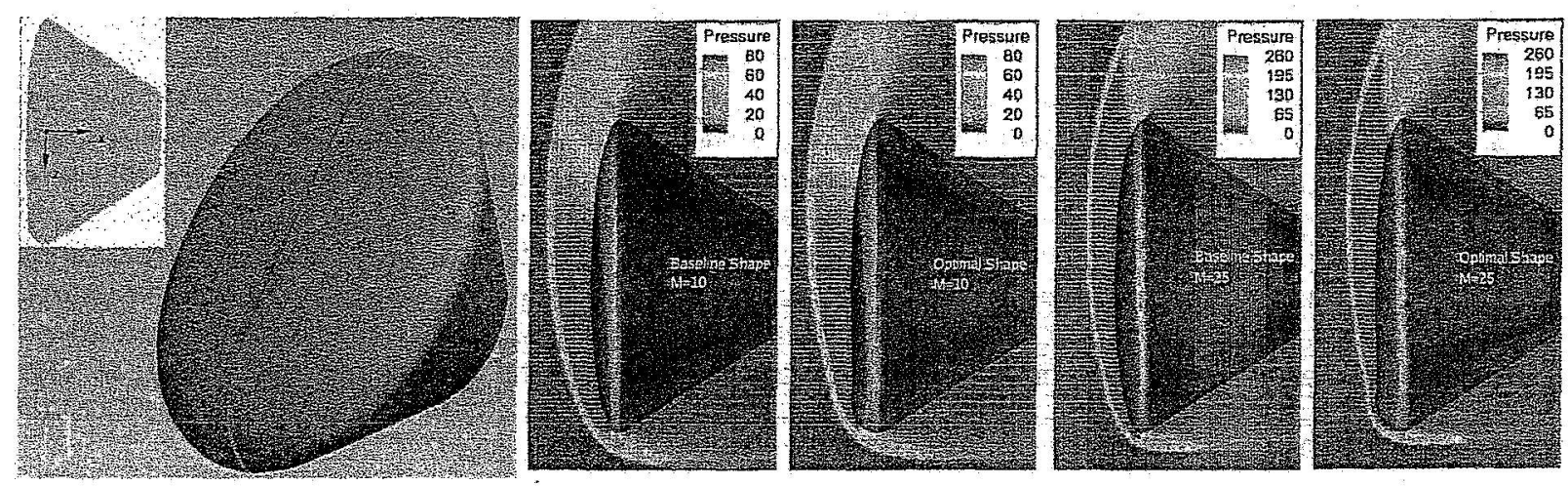

Fig. 1. Shape optimization of reentry capsule: Pro/ENGINEER CAD model (left image); pressure contours for the baseline and optimal shapes at $M=10$ and $M=25$ (center and right images, respectively).

\section{References}

1. Aftosmis, M. J., Berger, M. J., and Meiton, J. E., "Robust and Efficient Cartesian Mesh Generation for ComponentBased Geometry," AIAA Joumal, Vol. 36, No. 6, 1998, pp. 952-960.

2. Nemec, M., Aftosmis, M. J., Murman, S. M., and Pulliam, T. H., "Adjoint Formulation for an Embedded-Boundary Cartesian Method," AIAA Paper 2005-0877, Reno, NV, Jan. 2005.

3. Nemec, M. and Aftosmis, M. J., "Adjoint Algorithm for CAD-Based Shape Optimization Using a Cartesian Method," AIAA Paper 2005-4987, Toronto, ON, June 2005. 


\title{
Adjoint Sensitivity Computations for an Embedded-Boundary Cartesian Mesh Method and CAD Geometry
}

\author{
Marian Nemec ${ }^{1}$ and Michael J. Aftosmis ${ }^{2}$ \\ 1 ELORET Corp., Moffett Field, CA 94035, USA mnemec@mail.arc.nasa.gov \\ 2 NASA Ames Research Center, CA 94035, USA maftosmis@mail.arc.nasa.gov
}

Key words: shape optimization, adjoint, Cartesian mesh, cut-cells

\section{Introduction}

Complex geometry remains a challenging issue facing the application of adjoint and flow-sensitivity methods in practical engineering design. Among the most promising approaches for complex-geometry problems is the embedded boundary Cartesian-mesh method [1]. In this approach, the discretization of the surface geometry is decoupled from that of the volume mesh; which enables rapid and robust mesh generation, and ultimately, an automatic analysis of aerodynamic performance. The purpose of this work is to extend the automation and efficiency of Cartesian methods to the computation of aerodynamic sensitivities for shape optimization problems.

The most common way to account for the effect of boundary shape perturbations in the adjoint and flow-sensitivity formulations is via domain mapping approaches. This involves the use of mesh-perturbation schemes in conjunction with body-fitied structured or unstructured meshes. For local shape deformations, the extent of the mesh perturbations can be limited to just the boundary cells $[2,3,4]$. The approach we propose here for non-body-fitted Cartesian meshes is similar, but the boundary faces of the volume mesh maintain their Cartesian orientation as the surface evolves. This approach permits the computation of mesh sensitivities via a direct linearization of the boundary-cell geometric constructors of the mesh generator.

\section{Discrete Adjoints and Flow Sensitivities}

Our goal is to minimize a scalar objective function $\mathcal{J}$, such as drag, by adjusting a design variable $X$ using gradient-based optimization. To compute the gradient, $\mathrm{d} \mathcal{J} / \mathrm{d} X$, we use a discrete formulation. Hence, a variation in $X$ 
influences the computational mesh $M$ and the flow solution $Q$, which satisfies the three-dimensional Euler equations of a perfect gas. The spatial discretization of the flow equations uses a cell-centered, second-order accurate finite volume method with a weak imposition of boundary conditions, resulting in a system of equations

$$
R(X, M, Q)=0
$$

where $M$ is an explicit function of the surface triangulation $T: M=f[T(X)]$.

The gradient of the objective function $\mathcal{J}(X, M, Q)$ is given by

$$
\frac{\mathrm{d} \mathcal{J}}{\mathrm{d} X}=\frac{\partial \mathcal{J}}{\partial X}+\underbrace{\frac{\partial \mathcal{J}}{\partial M} \frac{\partial M}{\partial T} \frac{\partial T}{\partial X}}_{\mathrm{A}}+\frac{\partial \mathcal{J}}{\partial Q} \frac{\mathrm{d} Q}{\mathrm{dX}}
$$

The evaluation of the term $\mathrm{d} Q / \mathrm{d} X$, referred to as the flow sensitivities, is obtained by linearizing Eq. 1

$$
\frac{\partial R}{\partial Q} \frac{\mathrm{d} Q}{\mathrm{~d} X}=-(\frac{\partial R}{\partial X}+\underbrace{\frac{\partial R}{\partial M} \frac{\partial M}{\partial T} \frac{\partial T}{\partial X}}_{\mathrm{B}})
$$

The adjoint equation is obtained by combining Eqs. 3 and 2 and defining the following intermediate problem

$$
-\frac{\partial R^{T}}{\partial Q} \psi=\frac{\partial \mathcal{J}^{\mathrm{T}}}{\partial Q}
$$

where the vector $\psi$ represents the adjoint variables. Details of the solution method for Eqs. 3 and 4 are given in [5]. We focus on the evaluation of the terms $A$ and $B$ in Eqs. 2 and 3, which are discussed in the next section.

\section{Shape Sensitivities}

The flow equations are discretized on a multilevel Cartesian mesh. The mesh consists of regular Cartesian hexahedra everywhiere, except for a layer of bodyintersecting cells, or cut-cells, adjacent to the boundaries. An infinitesimal perturbation of the boundary shape affects only the cut-cells. The sensitivity of the residual equations, Eq. 1, to a variation in the surface shape requires the linearization of the Cartesian face areas and centroids, the wall normals and areas, and the volume centroids of the cut-cells. The crux of these computations is the linearization of the geometric constructors that define the intersection points between the surface triangulation and the Cartesian hexahedra.

We explain the salient steps of the linearization using the example shown in Fig. 1, where a Cartesian hexahedron is split into two cut-cells by the surface triangulation. We require the linearization of the intersection points 
that lie on Cartesian edges, e.g., point $A$, and also those that lie on triangle edges, e.g., point $B$. Focusing on point $B$, its location along the triangle edge $V_{0} V_{1}$ is given by

$$
B=V_{0}+s\left(V_{1}-V_{0}\right)
$$

where $s$ denotes the distance fraction of the face location relative to the vertices $V_{0}$ and $V_{1}$. The linearization of this geometric constructor is given by

$$
\frac{\partial B}{\partial X}=\frac{\partial V_{0}}{\partial X}+s\left(\frac{\partial V_{1}}{\partial X}-\frac{\partial V_{0}}{\partial X}\right)+\left(V_{1}-V_{0}\right) \frac{\partial s}{\partial X}
$$

A similar constructor is used for point $A[6]$. An example result of the linearization is shown in Fig. 1 for the position sensitivity of Cartesian face centroids. Note that the "motion" of the face centroids is constrained to the plane of the face. An advantage of this formulation is that the shape sensitivities of the triangulation, i.e., $\partial V_{1} / \partial X$ in Eq. 6 and the term $\partial T / \partial X$ in $\mathrm{Eq} .3$, are independent of the volume mesh sensitivities, $\partial M / \partial T$. Put another way, there is no requirement for a one-to-one triangle mapping as the surface geometry evolves. This allows a flexible interface for geometry control based on tools such as computer-aided design (CAD).

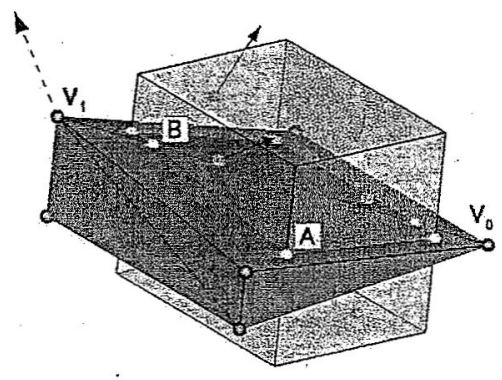

Fig. 1. Sensitivity of face centroids (solid vectors) to perturbation of vertex $V_{1}$.

\section{Verification Studies}

\subsection{Supersonic Vortex Problem}

We investigate the error convergence rate of a representative objective function and its gradient on a model problem with a known analytic solution [6]. The problem involves isentropic flow between concentric circular arcs at supersonic conditions, as shown in Fig. 2. The objective function is the integral of pressure along the outer arc, which is similar to the lift and drag objectives used in aerodynamic design. We compute the gradient and the sensitivities of the flow solution, Eq. 3, with respect to the inlet Mach number, $M_{i}$. The problem is solved on a sequence of five nested Cartesian meshes.

Figure 3 summarizes the results. Figure 3(a) shows the error convergence rates in the $\mathrm{L}_{1}$ norm of density and its sensitivity to variations in $M_{i}$. The error convergence rate of the objective function and its gradient is shown in Fig. 3(b). The asymptotic

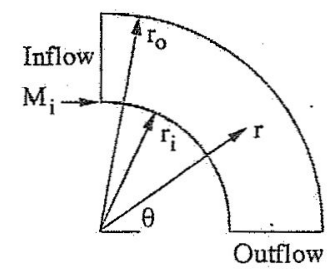

Fig. 2. Problem setup $\left(M_{i}=2.25, r_{i}=1\right.$ and $\left.r_{0}=1.382\right)$ 


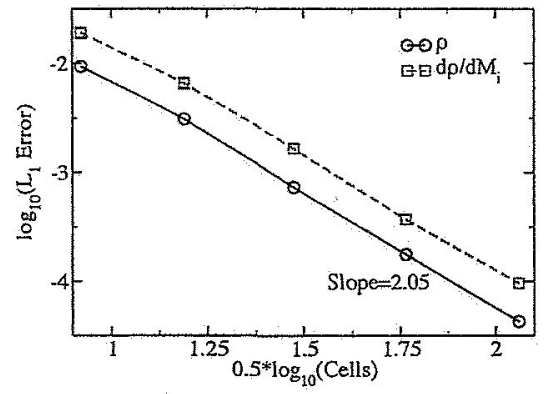

(a) Density and density gradient

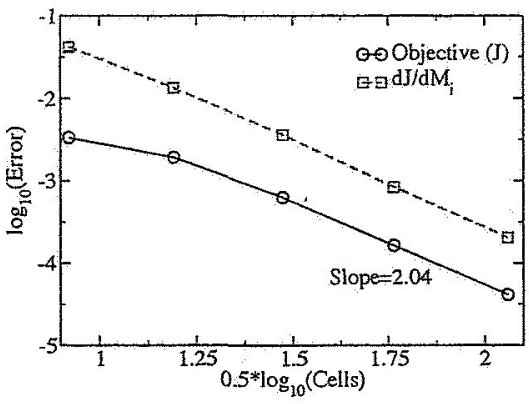

(b) Objective function and gradient

Fig. 3. Error convergence (slopes are computed using the three finest meshes)

convergence rate of all errors, which is measured over the three finest meshes, is just slightly over 2 . These results verify the accuracy of the linearization and the convergence of these methods to the continuous problem.

\subsection{Shape sensitivities for the NACA 0012 airfoil}

The objective of this verification test is to study the convergence of gradients for design variables that alter the shape of the boundary. We consider a subsonic flow over the NACA 0012 airfoil at $M_{\infty}=0.5$ and $\alpha=1 \mathrm{deg}$. We investigate the sensitivity of lift to the angle of attack using two equivalent approaches. First, we consider the influence of the angle of attack via a. change in the farfield boundary conditions, which does not alter the relationship between the mesh and the airfoil. We contrast this with an angle of attack change implemented via a rigid-body rotation of the airfoil about its trailing edge within a fixed mesh. The mesh-refinement study is performed on a sequence of five nested Cartesian meshes for each airfoil orientation.

The results are summarized in Fig. 4. Referring to Fig. 4(b), note that the differences in the gradients between the farfield and rigid-body rotation cases are decreasing as the mesh is refined. Additional regression analysis of this data indicates that the rate of convergence is first-order. This is a consequence of the fact that the mesh perturbations are confined to only the cut-cells. Unlike the supersonic vortex problem, a perturbation of the angle of attack via rigid-body rotation modifies the cut-cell boundary and introduces an error in the objective function proportional to second-order spatial discretization. Nevertheless, the gradient values for the rigid-body rotation case, even on the coarsest mesh of roughly 3,200 cells, are within $1 \%$ of the fine-mesh values.

\section{Design Example: Reentry Capsule}

We target the optimization of a heat-shield shape for a reentry capsule. The objective of the optimization is to enhance the lift-to-drag ratio, $L / D$, of 


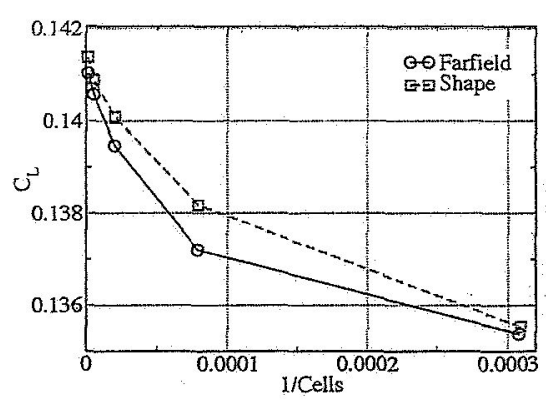

(a) Lift

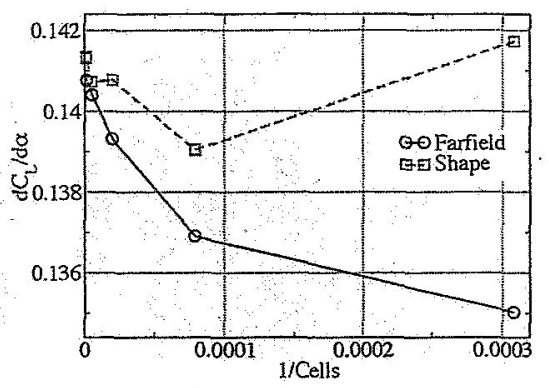

(b) Gradient of lift

Fig. 4. Convergence of lift and its gradient with respect to angle of attack: implemented via the farfield boundary ("Farfield") and rigid-body rotation ("Shape")

the capsule, thereby improving trajectory control for landing-site selection, and reducing the reentry load factor and heat rates. The Pro/ENGINEER ${ }^{\circledR}$ Wildfire CAD system is used to create the geometry model. The capsule configuration is shown in Fig. 5(a), where the design variables are the three labeled spline poirits of the heat-shield center-line. The freestream conditions are $M_{\infty}=10$ and $\alpha=156.5$ deg., measured clockwise from the positive $x$-axis. High-temperature effects are approximated by the use of an "effective" ratio of specific heats, $\gamma$. We use $\gamma=1.3$. The initial (symmetric) capsule generates an $L / D$ of 0.37 , which is attained using a center-of-gravity offset. The target value of $L / D$ is set to 0.4 . The volume mesh contains roughly 665,000 cells and we use 64 processors to solve the flow and adjoint equations.

Convergence of the optimization problem is shown in Fig. 5(b). The target $L / D$ is reached within five design iterations and the $L_{2}$ norm of the gradient is reduced by roughly four orders of magnitude. The initial and final heat-shield shapes are shown in Figs. 5(c) and 5(d), respectively. The shape modifications are relatively minor, yet the improvement in $L / D$ is $8 \%$. The wall-clock time per design iteration is approximately 11 minutes. This time includes the regeneration and triangulation of the part, as well as the flow solution and adjoint gradient computation. We emphasize that for problems with more design variables, the design-cycle time would remain essentially constant.

\section{Conclusions}

We have presented an approach for the computation of aerodynamic shape sensitivities using a discrete formulation on Cartesian meshes with cut-cells at the wall boundaries. The verification studies show that the convergence rate of gradients is second-order for design variables that do not alter the boundary shape, and is reduced to first-order for shape design variables. This is a consequence of confining the mesh sensitivities to the cut-cells. The design example demonstrates the effectiveness of the new approach for engineering design studies that require a fast turn-around and include CAD-based geometry, complex flow, and many design variables. 


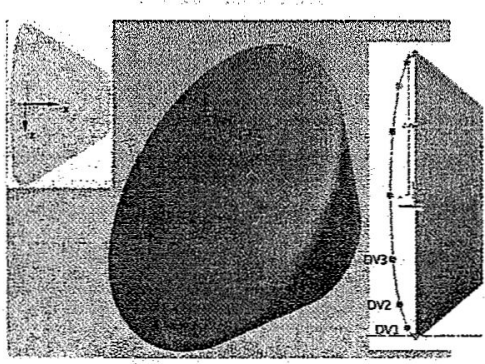

(a) CAD model

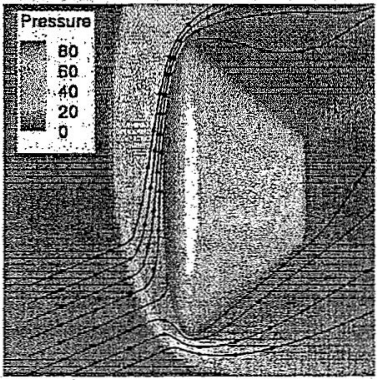

(c) Initial shape

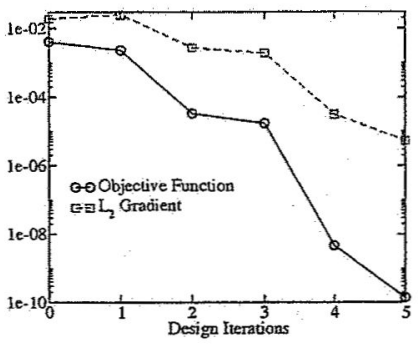

(b) Convergence history

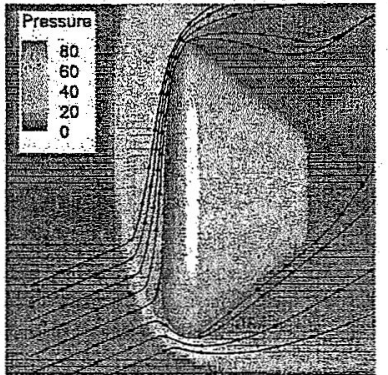

(d) Optimized shape

Fig. 5. Heat-shield shape optimization $\left(M_{\infty}=10, \alpha=156.5^{\circ}, \gamma=1.3\right)$

\section{Acknowledgments}

The authors gratefully acknowledge Robert Haimes (MIT) for help with CAPRI. This work was supported by the NASA Ames Research Center contract NNA05BF35C.

\section{References}

1. Aftosmis, M. J., Berger, M. J., and Melton, J. E., "Robust and Efficient Cartesian Mesh Generation for Component-Based Geometry," AIAA Journal, Vol. 36, No. 6, 1998, pp. 952-960.

2. Anderson, W. K. and Venkatakrishnan, V., "Aerodynamic Design Optimization on Unstructured Grids with a Continuous Adjoint Formulation," Computers 8 Fluids, Vol. 28, 1999, pp. 443-480.

3. Dadone, A. and Grossman, B., "Efficient Fluid Dynamic Design Optimization Using Cartesian Grids," AIAA Paper 2003-3959, Orlando, FL, June 2003.

4. Lu, J., An a posteriori Error Control Framework for Adaptive Precision Optimization using Discontinuous Galerkin Finite Element Method, Ph.D. thesis, Massachusetts Institute of Technology, 2005.

5. Nemec, M., Aftosmis, M. J., Murman, S. M., and Pulliam, T. H., "Adjoint Formulation for an Embedded-Boundary Cartesian Method," AIAA Paper 2005-0877, Reno, NV, Jan. 2005.

6. Nemec, M. and Aftosmis, M. J., "Aerodynamic Shape Optimization Using a Cartesian Adjoint Method and CAD Geometry," AIAA Paper 2006-3456, San Francisco, CA, June 2006. 